\title{
The Effect of Adding Magnesium to Bupivacaine as an Adjuvant in Continuous Pectoralis Nerve II (PECs) Block in Simple Mastectomy
}

\author{
Eman M Kamal Abo Seif, Walid Y Kamel and Amr M Hilal Abdou* \\ Department of Anesthesia, Ain Shams University, Egypt
}

*Corresponding author: Amr M Hilal Abdou, Department of anesthesia and intensive care. Ain Shams University, Egypt

\begin{abstract}
Background and Aim: Breast surgeries are associated with post-operative pain and restricted shoulder mobility, so the aim of this study is to assess the analgesic efficiency and safety of Continuous Pectorals II (PECs) block by adding magnesium to bupivacaine as an adjuvant versus bupivacaine as a sole agent in cases of simple mastectomy.

Patients and Methods: This randomized double blinded study designed to compare the analgesics efficacy and safety of continuous PECs II using bupivacaine versus continuous PEC II using bupivacaine plus magnesium in patients undergoing simple mastectomy and postoperative period. We enrolled 40 adult females ASA I-II patients' candidates for simple mastectomy without axillary clearance. All patients were randomly distributed into two groups. Control group: prior to surgical incision patients had ultrasound guided PEC II continuous block with $10 \mathrm{ml}$ plain bupivacaine $0.25 \%$ plus followed by infusion of $8 \mathrm{ml}$ normal saline per hour. Magnesium group: patients were given $10 \mathrm{ml}$ plain bupivacaine $0.25 \%$ plus $0.5 \mathrm{gm}(5 \mathrm{ml})$ magnesium sulfate followed by

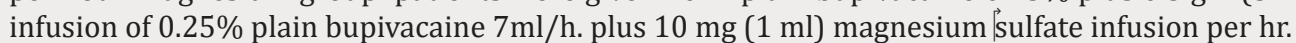

Results: For assessment of the postoperative pain scores by the VAS; our results showed significant reduction in the magnesium group $(\mathrm{P}<0.001)$ in comparison with control group. Intraoperative vital data in the form of heart rate $(\mathrm{HR})$ and mean arterial blood pressure (MAP) showed highly significant decrease of the heart rate in the magnesium group $(\mathrm{P} \leq 0.001)$ after 30 minutes from the block till 2 hours after the block compared to the control group, in addition to significant decrease in MAP $(\mathrm{P}=0.036)$ after the block by 30 minutes. Postoperative vitals showed significant decrease of HR $(P=0.016)$ in magnesium group 4 hours after surgery till the following day, highly significant decrease in RR ( $\mathrm{P}<0.001)$.

Conclusion: This clinical study proven that mixing of magnesium sulphate to bupivacaine during PECs II block provided a solid safe prolonged motor and sensory block, besides the reduction of postoperative additional rescue pain medications and pain scores.

\section{Introduction}

Acute postoperative pain and shoulder immobility is a usual complication following breast surgeries. Many technique are used to reduce the postoperative pain, thoracic paravertebral block (TPVB) is the most widely used technique to provide postoperative analgesia but it does not block medial and lateral pectoral nerves effectively with risk of spinal cord trauma, pneumothorax and sympathetic block [1]. Blocking the pectoral nerve (PEC I) provides intraoperative and postoperative pain control during breast surgery. The Pec I block is a superficial block that has been used effectively. The PECs II block is more effective and used widely now because it blocks also the long thoracic and the thoraco-dorsal nerves in addition to the lateral branches of the intercostals nerves that present at the level of the mid axillary line innervating mammary gland and T2 to T6 overlying skin [2]. To achieve adequate PECs II block Local anesthetic should be infiltrated under the Pectoralis minor muscle between the Clavi-pectoral fascia and the superficial border of the serratus muscle thus covering the pectoral region with the pectoral nerves and the intercostals branches [3].The block is done in supine position and the arm either abducted 90 degrees or by the patient's side. Injecting the anterior axillary line at the level of the fourth rib 3-6 cm in depth with the transducer of the 
ultrasound machine at the mid clavicular level and angled inferolaterally after identifying the axillary artery, vein and the second rib [4]. The transducer then is moved lateral until the Pectoralis minor and serratus anterior are identified, the local anesthetic is injected between the two muscles. For continuous PECs II bock, a catheter is inserted in the fascial plane and positioned to ensure intraoperative and postoperative pain control [5].

Bupivacaine is an amide local anesthetic with a slow onset, long duration of action and high potency. Bupivacaine has a toxicity profile among the worst of the amide local anesthetic, the maximum recommended single bolus dose of bupivacaine is 2.5 $\mathrm{mg} / \mathrm{Kg}$, it is metabolized in the liver by human cytochrome P450 (CYP) and the metabolites are non-toxic by the CYP3A4 in an oxidative metabolism fashion. Bupivacaine exert its action through impairing sodium influx across the neuronal membrane by blocking the sodium channels. Magnesium is the second most common intracellular cation after potassium. It acts as co-factor in more than 300 enzymatic reactions and also involved in several processes as neurotransmitter release, cardiac excitability, neuronal activity and control or vasomotor tone [6]. The total normal serum $\mathrm{Mg}+2$ levels is in the range of 1.8 to $3 \mathrm{ml} / \mathrm{dl}$. The sole route of elimination of $\mathrm{Mg} 2+$ is by renal clearance. Magnesium has a depressant action on the central nervous system and has been used as an anticonvulsant [7]. The mechanism of action is related to competition with calcium in the processes of transmitter release which leads to pre-synaptic inhibition of acetylcholine release at the neuromuscular function. It also exerts its analgesic action through blocking N-methyl-D aspirate (NMDA) receptors [8]. Mg2+ potentiates the action of local anesthetics (LA) in the peripheral nerves by elevating the firing threshold in both myelinated and unmyelinated axons increasing the transmembrane potential causing hyperpolarization [9] addition of magnesium to bupivacaine can result in an enhancement of nerve blocking.

\section{Methodology}

Following approval from our faculty ethical committee and obtained written informed consent from the patients, we enrolled 40 adult females ASA I-II scheduled for simple mastectomy in this randomized double blinded study. All patients were randomly assigned to one of two groups. Patients known to be allergic to local anesthetics, or diagnosed with coagulopathy or on anticoagulant therapy, or having skin infection at the injection site are excluded from this study. Control group: prior to surgical incision patients had ultrasound guided continuous PECs II block and injected with $10 \mathrm{ml}$ plain bupivacaine $0.25 \%$ plus $8 \mathrm{ml}$ normal saline per hr. Magnesium group; Patients had ultrasound guided continuous PECs II block and injected $10 \mathrm{ml}$ plain bupivacaine $0.25 \%$ plus $0.5 \mathrm{gm}(5 \mathrm{ml})$ magnesium sulphate, followed by infusion of $0.25 \%$ plain bupivacaine $7 \mathrm{ml} / \mathrm{hr}$. plus $10 \mathrm{mg}(1 \mathrm{ml})$ magnesium sulphate infusion per hour.

\section{Anesthetic Management}

History and preoperative assessment were done for all patients of the two groups. Full preoperative profile with coagulation investigations was done. Patient education on evaluation of the intensity of pain via the visual analog scale (VAS), scored from 0-10 (where $0=$ no pain and $10=$ worst pain). All patients had the same premedication such as Midazolam $0.1 \mathrm{mg} / \mathrm{kg}$. Standard monitors were applied for all patients. Conventional general anesthesia with Propofol and fentanyl 1- mcg/kg and atracurium $0.5 \mathrm{mg} / \mathrm{kg}$ were standardized for all patients.

\section{PECs II Block Technique}

While patient is in supine position either with abducted arm 90 degrees or beside the patient. Injection done at fourth rib's level anterior axillary line, under ultrasound guidance (Sonosite M-Turbo, USA) The depth was adjusted to 3-6 $\mathrm{cm}$ with the transducer at the midclavicular level and angled infero-laterally the transducer was then moved laterally to identify the Pectoralis minor and serratus anterior. The local anesthetic was injected between the last two muscles, followed by insertion of a B Braun catheter kit Periflix (B. Braun Melsungen AG, Germany) with an 18 gauge needle in the fascial plane was done to insure continuous PECs II block for the operative and postoperative pain control. Intraoperative analgesic control was maintained by Fentanyl $1 \mathrm{mcg} / \mathrm{kg}$ if there was heart rate or blood pressure elevation more than $20 \%$ of the baseline, concerning the postoperative analgesia VAS was assessed first if more than 5 ; Morphine bolus $0.1 \mathrm{mg} / \mathrm{kg}$ was given.

\section{Collected Data}

Heart rate and mean blood pressure were assessed before induction of anesthesia then 15 minutes after the block then every 15 minutes till the end of surgery, respiratory rate values were recorded for the first 24 hours postoperatively every 2 hours, all the previous variables were added to the VAS score, time of first analgesic dose and total morphine dosage given for the first $24 \mathrm{hr}$ after surgery.

\section{Statistical Analysis}

For each patient, results were calculated from baseline values and were considered for comparison between the groups. All results are described as mean \pm SE. comparison were made using the student's t-test, the Mann-Whitney U-test or Chi square test. The threshold for statistical significance was taken as $\mathrm{P}<0.05$. Randomization was done using a computer-generated sequence. Concealment was achieved using opaque envelopes.

\section{Results}

Forty adult females 31 of them were ASA I, 9 of them were ASA II were recruited for simple mastectomy then randomly divided into control group and magnesium group of average duration (Mean \pm SD) of surgery $131.5 \pm 6.53$ and $127.1 \pm 7.35$, The demographic data Table 1 as regards age, weight, Duration of surgery and ASA score showed no significant difference $(\mathrm{P}=0.167$, $0.578,0.057$, and 0.705$)$ respectively. $2(10 \%)$ cases in the control group required intraoperative fentanyl doses and no $(0.0 \%)$ cases took any fentanyl in the magnesium group. Intraoperative vital data in the form of heart rate (HR) and mean arterial blood pressure (MAP) (Figures 1\&2) showed highly significant decrease of the heart rate in the magnesium group $(\mathrm{P} \leq 0.001)$ after 30 minutes from the block till 2 hours after the block compared to the control 
group, in addition to significant decrease in MAP $(\mathrm{P}=0.036)$ also 30 minutes after the block. Postoperative vitals Table 2 showed significant decrease of HR $(\mathrm{P}=0.016)$ in magnesium group 4 hours after surgery till the following day, highly significant decrease in RR ( $\mathrm{P}<0.001$ ) with no detected complications such as ICU admission or Oxygen therapy, SPO2 measurements were normal in any group

Table 1: Demographic data of patients included in the study. for the first day following surgery. With respect to the postoperative MAP there was highly significant $(\mathrm{P}=0.001)$ decrease towards the magnesium group. For assessment of the postoperative pain scores by the VAS; our results (Figure 3 ) showed highly significant decrease in the magnesium group $(\mathrm{P}<0.001)$ when compared to the control group.

\begin{tabular}{|c|c|c|c|c|c|c|}
\hline \multicolumn{2}{|c|}{} & Control Group & Magnesium Group & Test Value & P-Value & Sig. \\
\hline Age & Mean \pm SD & $39.32 \pm 10.18$ & $43.15 \pm 6.67$ & 1.407 & 0.167 & NS \\
\hline Weight $(\mathrm{kg})$ & Mean \pm SD & $75.32 \pm 6.75$ & $74.16 \pm 6.24$ & 0.564 & 0.578 & NS \\
\hline Duration (min) & Mean \pm SD & $131.5 \pm 6.53$ & $127.18 \pm 7.35$ & 1.965 & 0.057 & NS \\
\cline { 1 - 5 } ASA score & ASA I & $16(80.0 \%)$ & $15(75.0 \%)$ & \multirow{2}{*}{0.143} & \multirow{2}{*}{ NS } \\
\cline { 2 - 5 } & ASA II & $4(20.0 \%)$ & $5(25.0 \%)$ & & \\
\hline
\end{tabular}

Table 2: Postoperative heart rate values and MAP values for both groups.

\begin{tabular}{|c|c|c|c|c|c|}
\hline & Control Group & Magnesium Group & Test Value & P-Value & Sig. \\
\hline \multicolumn{6}{|c|}{ HR } \\
\hline 4 hours & $74.18 \pm 5.23$ & $69.13 \pm 7.32$ & 2.51 & 0.016 & $\mathrm{~S}$ \\
\hline 6 hours & $73.39 \pm 5.12$ & $67.45 \pm 7.65$ & 2.886 & 0.006 & HS \\
\hline 12 hours & $72.13 \pm 4.98$ & $65.24 \pm 7.54$ & 3.41 & 0.002 & HS \\
\hline 24 hours & $71.36 \pm 5.64$ & $63.09 \pm 7.83$ & 3.833 & 0.001 & HS \\
\hline \multicolumn{6}{|c|}{$\mathbf{R R}$} \\
\hline 4 hours & $11.20 \pm 0.56$ & $10.36 \pm 0.51$ & 4.96 & $<0.001$ & HS \\
\hline 6 hours & $10.83 \pm 0.45$ & $10.09 \pm 0.42$ & 5.376 & $<0.001$ & HS \\
\hline 12 hours & $10.56 \pm 0.42$ & $9.13 \pm 0.43$ & 10.639 & $<0.001$ & HS \\
\hline 24 hours & $10.30 \pm 0.65$ & $9.05 \pm 0.33$ & 7.669 & $<0.001$ & HS \\
\hline \multicolumn{6}{|c|}{ MAP } \\
\hline 4 hours & $74.13 \pm 8.25$ & $65.12 \pm 7.32$ & 3.653 & 0.001 & HS \\
\hline 6 hours & $73.22 \pm 7.68$ & $64.19 \pm 7.50$ & 3.762 & 0.001 & HS \\
\hline 12 hours & $72.03 \pm 7.51$ & $61.82 \pm 7.21$ & 4.386 & $<0.001$ & HS \\
\hline 24 hours & $71.10 \pm 7.13$ & $65.20 \pm 7.58$ & 2.536 & 0.016 & $S$ \\
\hline
\end{tabular}

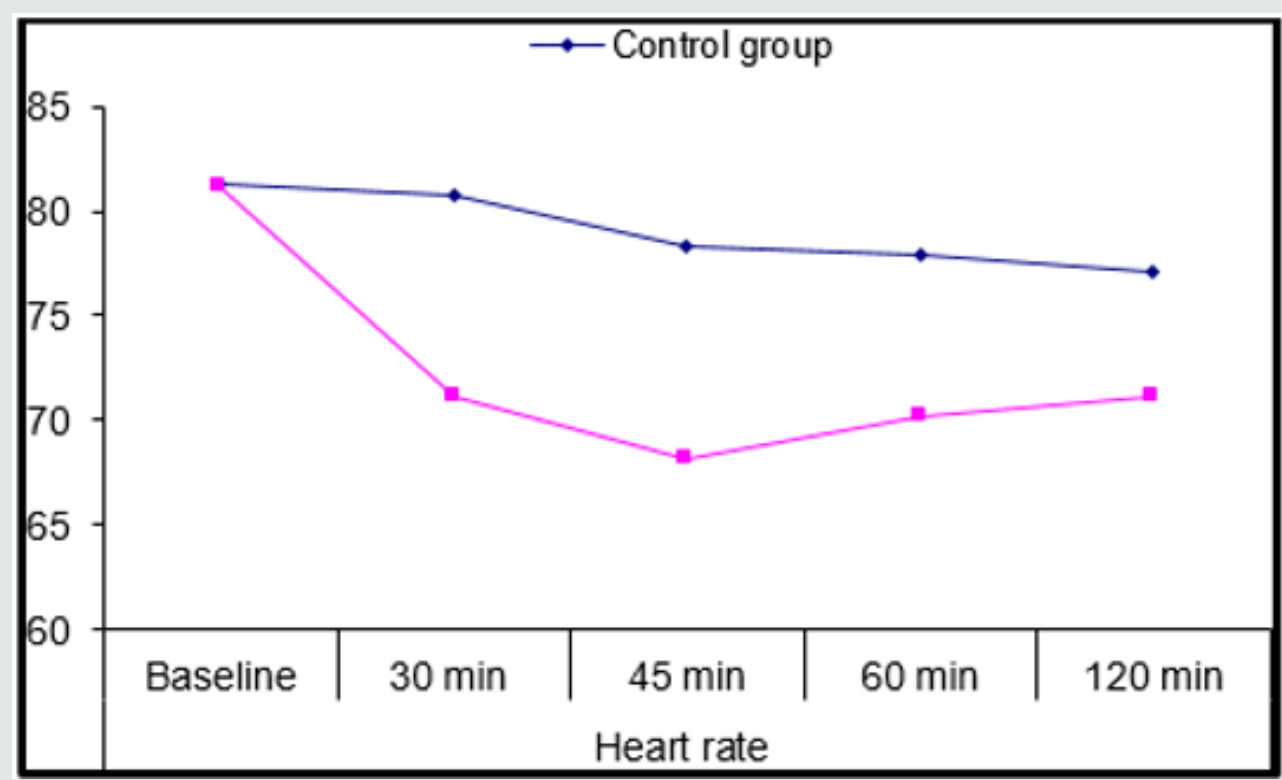

Figure 1: Intraoperative heart rate values for both groups. 


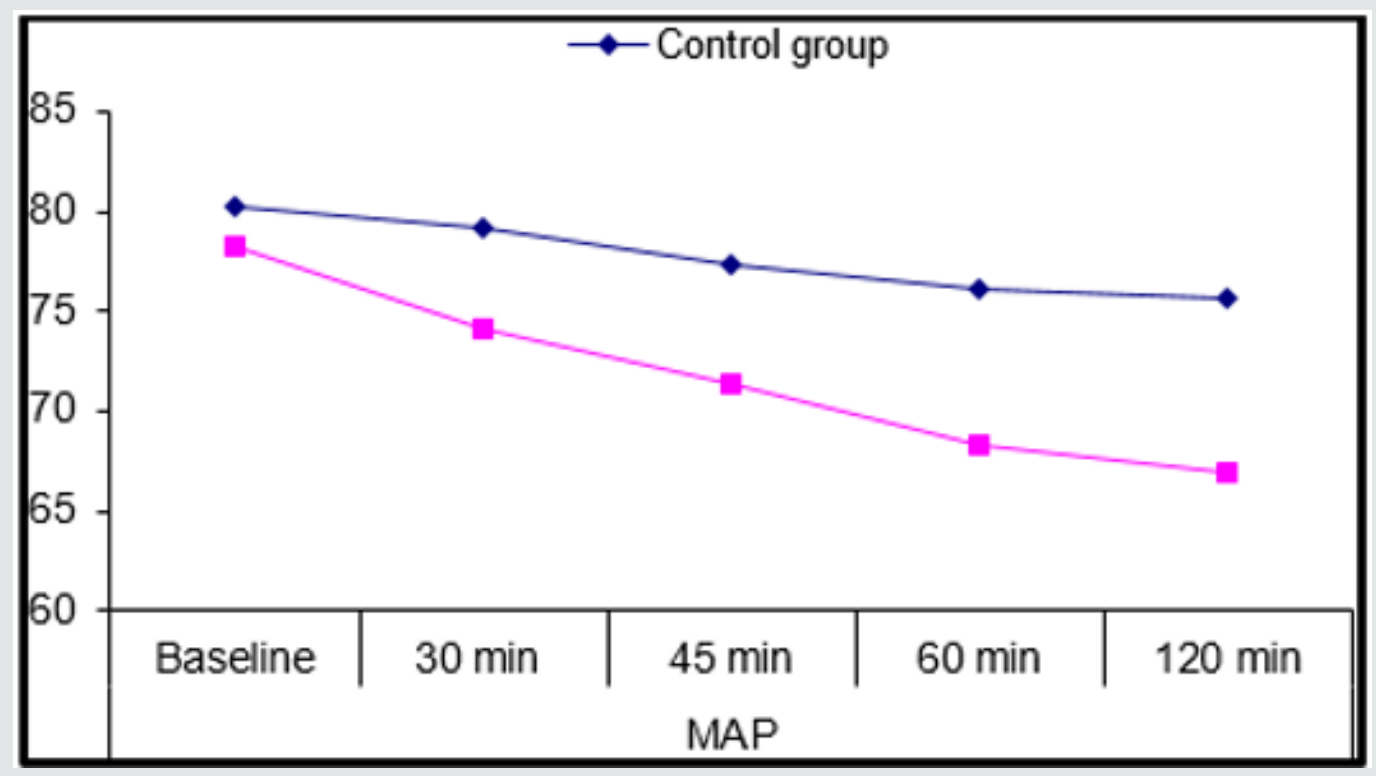

Figure 2: Intraoperative MAP values for both groups.

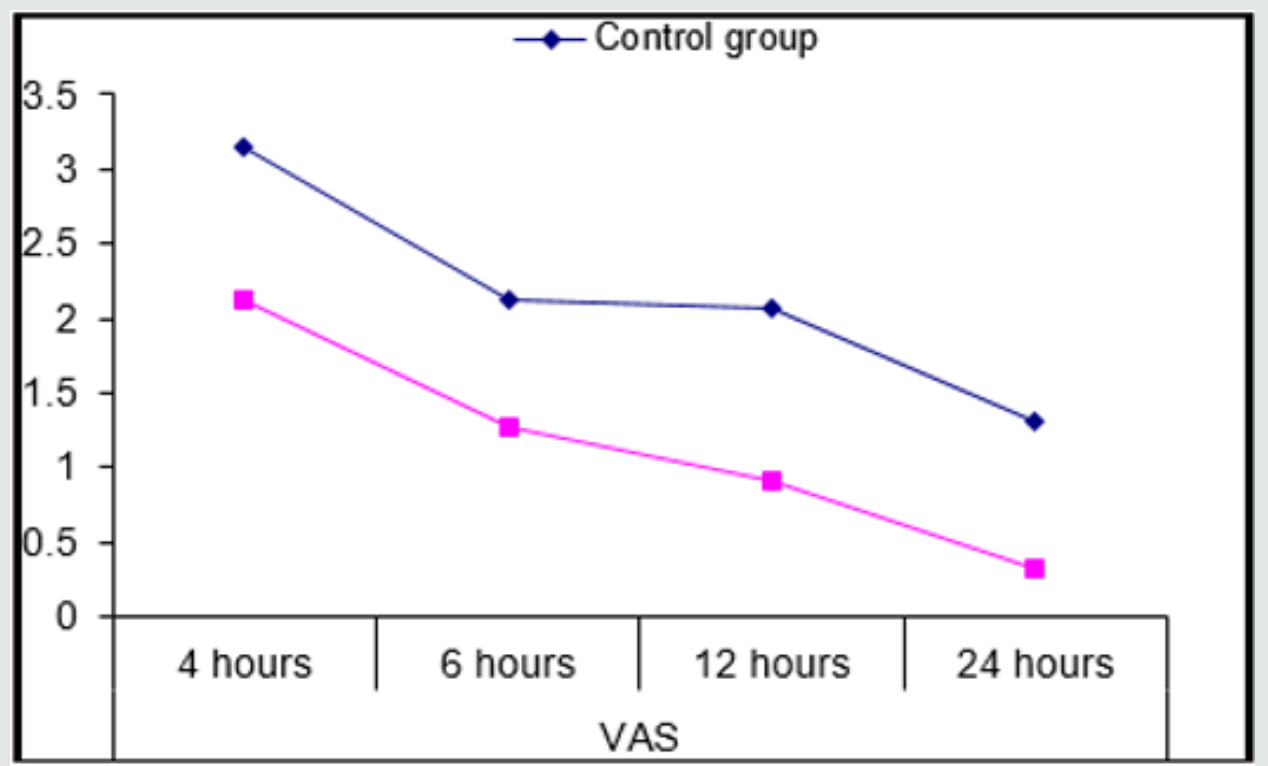

Figure 3: Postoperative VAS values for both groups.

\section{Discussion}

Many types of regional methods such as intercostal nerve blocks, epidural block and paravertebral nerve block have been used for controlling postoperative pain following breast surgeries [10]. Pectoral nerve block is a technique first done by Blanco in 2011; after one year he modified the block into a second version (Modified peck block=PECs II) aiming to block the axilla and the intercostals nerve [11]. This study compared the analgesics effectiveness and safety of continuous PECs II using bupivacaine versus continuous PECs II using bupivacaine plus magnesium in patients had breast lumpectomy both for pain control during and after the surgery. The results showed that there was a good control for intra and postoperative data regarding hemodynamics (mean arterial blood pressure and pulse rate), the results also showed a decrease in intraoperative consumption of fentanyl. Postoperative score of pain was more in the control group comparing to the magnesium group, and none of the patients requested for rescue analgesia in the postoperative day. In contrast to the results of this study, Hung et al. [12] studied the magnesium sulfate effect on amide LA in sciatic nerve block and concluded that magnesium (Mg) shortens the sciatic nerve block duration so it does not seem to be useful as adjuvant in peripheral nerve block. However, Bondok and her colleagues [13] studied the effect of injection of magnesium in the knee in arthroscopy procedure, and proved that this technique resulted in a significant reduction in the postoperative VAS in the first $24 \mathrm{hrs}$ in addition to significant decrease in the 
dose of postoperative analgesics. Furthermore, Kashedi et al. [14] compared the effect of analgesia of Mg plus Lidocaine, paracetamol plus Lidocaine and placebo plus Lidocaine on block characteristic for intravenous regional anesthesia (IVRA) in patients undergoing upper extremity orthopedic surgery: it was found that time from drug injection to sensory block onset was the shortest in the magnesium plus Lidocaine group. The duration of the motor block was prolonged in the Lidocaine plus Mg group.

\section{Conclusion}

This clinical study proven that mixing of magnesium sulphate to bupivacaine during PECs II block provided a solid safe prolonged motor and sensory block, besides the reduction of postoperative additional rescue pain medications and pain scores.

\section{Conflict of interest}

Nothing to disclose.

\section{Author's Contribution}

We Dr E.K.A/A.M.A/W.Y.K hereby declare that the article has not been published or submitted to or accepted for publication in any form in any other journal. I vouch that the authorship of this manuscript will not be contested by anyone whose names are not listed. On acceptance the article will become the copyright of Journal. All Authors has read and approved this manuscript.

\section{References}

1. Lin N, Yu B, Hung C (2016) Paravertebral Block Plus Thoracic Wall Block Versus Paravertebral Block Alone for Analgesia of Modified Radical Mastectomy: A Retrospective Cohort Study. PLoS One 11(11): e0166227.

2. Kulhari S, Bharti N, Bala I, Arora S, Singh G (2016) Efficacy of Pectoral Nerve Block Versus Thoracic Paravertebral Block for Postoperative
Analgesia After Radical Mastectomy: A Randomized Controlled Trial. BJA 117(3): 382-386.

3. Womack J, Varma M (2014) Seratus Plane Block for Shoulder Surgery. Anaesthesia 69(4): 395-396.

4. fujiwara A, Komasawa n, Minami T (2014) Pectoral Nerves (pecs) and Intercostals Nerve Block for Cardiac Resynchronization Therapy Device Implantation. Springerplus 3: 409.

5. Blanco R, Fajardo M, Maldonado T (2012) Ultrasound Description of PECs II (Modified Pecs I): Noval Approach to Breast Surgery. Rev Esp Anestesiol Reanim 59(9): 470-475.

6. Ramamurthi R, Krane J Elliot (2007) Local Anesthetic Pharmacology in Pediatric Anesthesia. Techniques in Regional Anesthesia and Pain Management 11(4): 229-234.

7. Dube L, Granry J (2003) The Therapeutic Use of Magnesium in Anesthesiology, Intensive Care and Emergency Medicine: A Review. Can J Anesth Can D Anesthésie 50(7): 732-746.

8. Blaine J, Chonchol M, Levi M (2015) Renal control Of Calcium Phosphate and Magnesium Homeostasis. Clin J Am Soc Nephrol 10(7): 1257-1272.

9. de Baaij J, Hoenderop J, Bindels R (2012) Regulation of Magnesium Balance: Lessons Learned from Human Genetic Disease. Clin Kidney J 5: i15-i24.

10. Song W, Chang Y (2012) Magnesium Sulfate for Acute Asthma in Adults: A Systematic Literature Review. Asia Pac Allergy 2(1): 76-85.

11. Lynch E, Welch K, Caraduena J (1995) Thoracic Epidural Anesthesia Improves Outcome After Breast Surgery. Ann Surg 222: 663-669.

12. Hung Y, Chen C, Lirk P, Wang CF, Cheng JK et al. (2007) Magnesium Sulphate Diminishes the Effects of Amide Local Anesthetics in Rat Sciatic Nerve Block. REG Anesth Pain Med 32: 288-295.

13. Bondok R, Abd El-Hady A (2006) Intra-Articular Magnesium Is Effective for Postoperative Analgesia in Arthroscopic Knee Surgery. Br j anesth 97(3): 389-392.

14. Kashefi P, Montazeri K (2008) Adding Magnesium Sulphate to Lidocaine of Intravenous Regional Anesthesia. Jrnl Res Anesht Pain Med 33(5): 97.
This work is licensed under Creative Commons Attribution 4.0 License

To Submit Your Article Click Here: Submit Article

DOI: $10.32474 /$ GJAPM.2020.02.000148

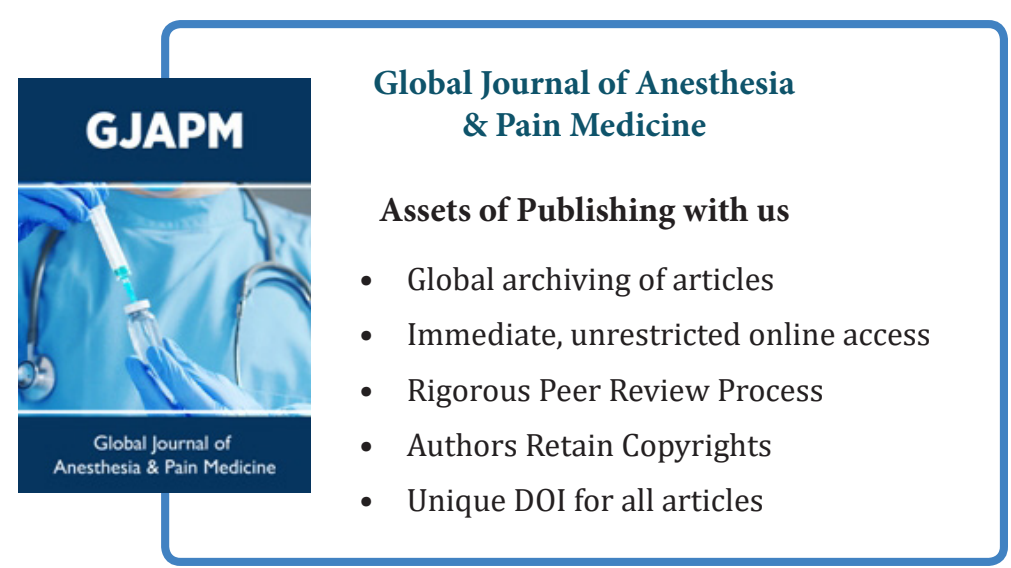

